\title{
Transition to turbulent heat transfer in heated vertical channel - Scaling analysis
}

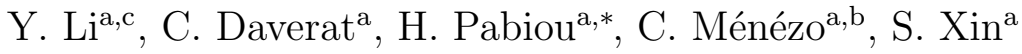 \\ ${ }^{a}$ Univ Lyon, CNRS, INSA-Lyon, Universit Claude Bernard Lyon 1, CETHIL UMR5008, \\ F-69621, Villeurbanne, France \\ ${ }^{b}$ Chaire INSA/EDF, Habitats et Innovations Energétiques, Lyon, France \\ ${ }^{c}$ Agence de l'Environnement et de la Maîtrise de l'Énergie 20, avenue du Grésillé- BP \\ 9040649004 Angers Cedex 01 France
}

\begin{abstract}
A thermally driven flow in a vertical channel with heated walls is studied through a scaling analysis and results are compared with the experimental data reported in [1]. As the geometry is symmetrical with respect to the vertical axis, the half-channel is considered. The domain is split into seven zones in which the flow behaviour results from a balance between several phenomena. This zonal analysis exhibits the main phenomena that drive the flow in each zone. The study leads to an expression of the wall temperature profile below and above the transition. An expression is given for the driving pressure that is found to increase along the channel. The increase rate is much more important above the transition due to the fluid heating in the center of the channel. Finally, The maximum Reynolds stress is expressed in terms of the mean velocity values and is found to fit well with the experimental data.

Keywords: Natural convection, turbulent heat transfer, vertical opened channel, scaling analysis

PACS: 47.55.pb, 44.25.+f, 44.15.+a, 47.27.te
\end{abstract}

\section{Introduction}

Natural convection in vertical channels has attracted a large numbers of researches due to its applications to cooling system of electronic components

\footnotetext{
${ }^{*}$ Corresponding author

Email address: herve.pabiou@insa-lyon.fr (H. Pabiou)
} 
or to thermally driven energy components in buildings. For example, photovoltaic double-façades are composed of a vertical photovoltaic wall separated from the primary envelope of the building by an opened air gap where natural convection occurs. Understanding heat transfer inside the double-façade is a challenge for energy management in the building. Such a complex component can be modelled by a vertical channel heated on one wall, the other one being adiabatic and located in an isothermal and infinite surrounding. Two important characteristics of such a configuration have to be noticed. Firstly, this flow is characterized by vertical isothermal lines near the heated wall that are parallel to the direction of the buoyant force. Therefore, there is no zero-velocity solution for the flow. Secondly, this open flow is characterized by a mean flow rate, the prediction of which is of great interest for engineering applications.

An experimental study carried out by the authors on a similar configuration characterized by a vertical channel in water with symmetrical heating, has been first reported in [2]. This study has led to a more detailed experimental characterization reported in [1] which is also referred in this article as the companion paper or the experimental study. The aim of the present study is to provide a scaling analysis of this experimental study. Indeed, numerous experimental or numerical data for such configuration are given in the scientific literature (see [1], [2] and references therein) but few of them deal with scaling analysis whereas it is a powerful tool to understand physical phenomena that drive a flow. Indeed, such analysis have already been used to study other classical thermally driven flows: the heated vertical plane plate and the Rayleigh-Bénard flow.

Concerning the thermally driven laminar flow along a vertical heated plate with isoflux condition, a self-similar solution is given by Sparrow and Gregg [3]. The wall temperature relative to the ambient and the thermal boundary layer thickness are found to be proportional to the distance to the leading edge to the power of $1 / 5$, whereas velocity is found to follow a $3 / 5$ power law. These power laws are easily retrieved by a scaling analysis as proposed by Lin and Armfield [4] that exhibits a steady state solution for Prandtl numbers $(P r)$ lower than unity. For Prandtl numbers larger than one, Lin, Armfield et al [5] have developed a similar analysis for a vertical plate heated at a uniform temperature. In these studies, the viscous boundary layer is defined as the zone where vertical velocity is not negligible and 
it is split into inner- and outer- layers with respect to the location of the velocity peak. To our knowledge, there has been no such scaling analysis for turbulent flow along a vertical plate. A similar analysis by Khanal and Lei [6] has been derived for a laminar thermally driven flow in a vertical channel. A vertical channel with one wall heated at a constant heat flux is analysed through a scaling analysis of the 2D-equations of conservation. In this study, the theory developed by Lin et Armfield [4] is adapted to the channel configuration. Two regimes are considered depending on whether the thermal boundary layers are distinct or not, the later case corresponding to very low Rayleigh numbers. For the distinct thermal boundary layers case and for the steady-state regime, the solution found by Lin and Armfield is retrieved. Hence this result is consistent with the analysis of Bar-Cohen and Rohsenow [7] that showed a good agreement of the experimental data by Sobel et al. [8] with the self-similar solution given by Sparrow and Gregg [3].

By contrast, a lot of scaling analysis have been developed for turbulent convection in the Rayleigh-Bénard configuration (see for example [9] [10]). In particular, Grossmann and Lohse [11] [12] have proposed a theory based on scaling analysis to obtain relationship between dimensionless numbers. Applied to Rayleigh-Bénard configuration, the so-called GL-theory considers the viscous and thermal turbulent dissipation rates in the whole flow as the sum of dissipation rates evaluated in the thermal boundary layer and in the bulk flow. It results in relationships between Nusselt, Rayleigh and Reynolds numbers that are the sum of two power laws. In these studies, the thermal boundary layer that is defined by the wall-normal temperature gradient, is supposed to be laminar. The scales of the thermal- and viscous- boundary layer thickness follow the classical Blasius law meaning that they are proportional to the Reynolds number to the power of $-1 / 2$, the Reynolds number being based on the height of the cavity and on the wind velocity ([11]). This Blasius law also applied to the boundary layers thickness of the laminar flow in the vertical plate configuration ([5]). Indeed, in the energy equation the balance between the vertical transport of heat by convection and the horizontal conduction of heat leads directly to the Blasius scaling law. Recently, $\mathrm{Ng}$ et al [13] applied the GL-theory to thermally driven flow in a 2D vertical channel heated on one plate and cooled on the other one. The temperature of the plates are uniform and the channel is considered as infinitely long. Using 2D direct numerical simulation, they showed that the velocity and temperature scales has to be different in the boundary layers and in the bulk 
flow to verify the GL-theory. The determination of relationships between these scales has to be found out in order to obtain global Nusselt Rayleigh numbers relationship.

In this study, a scaling analysis of a thermally driven flow in a vertical channel with symmetrically heated plates at a constant heat flux is carried out. Considering that the height of the channel is much larger than its width and depth, the flow is studied through 2D boundary layer like equations. The analysis is based on the partition of the half-channel in 7 zones, each zone being characterized by specific scales. The hypothesis are verified by the comparison with experimental data reported in the companion paper. These experimental data correspond to a modified Rayleigh number: $R a^{*}=$ $\frac{g \beta q_{\mathrm{w}} b^{5}}{\lambda \nu \kappa H}=6.710^{7}$ (for notations see the nomenclature). The article is organized as follows: the governing equations are given in Section 2 and the partition of the domain is described in Section 3. In Section 4, a zonal analysis is conducted. The flow is analysed in terms of heat transfer, pressure and Reynolds stress evolution along the channel. Some results are discussed in Section 5 .

\section{Nomenclature}

$b \quad$ Channel width $(\mathrm{m})$

$c_{f g} \quad$ Correlation coefficient for the quantities $f$ and $g$

$f(x) \quad$ Function defined by Eq.23 $\left(\mathrm{m}^{2} \mathrm{~s}^{-2}\right)$

$g \quad$ Acceleration of gravity $\left(\mathrm{m} \mathrm{s}^{-2}\right)$

$H \quad$ Channel height $(\mathrm{m})$

$h^{\prime}(x, y) \quad$ Function defined by Eqs.19 and 20, $\left(\mathrm{m}^{2} \mathrm{~s}^{-2}\right)$

$I_{\text {u,entry }} \quad$ Turbulent intensity at the entry

$l \quad$ Channel depth $(\mathrm{m})$

$P \quad$ Mean pressure, $\mathrm{Pa}$

$P_{g} \quad$ Mean driving pressure, $P_{g}=P+\rho_{\text {inlet }} g x, \mathrm{~Pa}$

$Q_{\text {conv, i }}$ Vertical heat rate in Zone i per unit of depth $\left(\mathrm{W} \mathrm{m}^{-1}\right)$

$Q_{\text {conv, v }}^{\prime}$ Horizontal heat rate at the boundary V-VI per unit of depth $\left(\mathrm{W} \mathrm{m}^{-1}\right)$ 


\begin{tabular}{|c|c|}
\hline$q_{w}$ & Wall heat flux $\left(\mathrm{W} \mathrm{m}^{-2}\right)$ \\
\hline$R a^{*}$ & Modified Rayleigh number, $R a^{*}=\frac{g \beta q_{\mathrm{w}} b^{5}}{\lambda \nu \kappa H}$ \\
\hline$T_{c}(x)$ & Mean temperature at the channel centre $(\mathrm{K})$ \\
\hline$T_{\text {ref }}$ & $\begin{array}{l}\text { Reference temperature for the Boussinesq approxima- } \\
\text { tion }(\mathrm{K})\end{array}$ \\
\hline$U, V, W$ & $\begin{array}{l}\text { Mean velocity component in the directions } x, y, z \text {, re- } \\
\text { spectively }\left(\mathrm{m} \mathrm{s}^{-1}\right)\end{array}$ \\
\hline$u^{\prime}, v^{\prime}, w^{\prime}$ & $\begin{array}{l}\text { Instantaneous velocity fluctuations in the directions } \\
x, y, z \text {, respectively }\left(\mathrm{m} \mathrm{s}^{-1}\right)\end{array}$ \\
\hline$u^{*}, v^{*}, w^{*}$ & $\begin{array}{l}\text { Order of magnitude of the velocity fluctuations in the } \\
\text { directions } x, y, z \text {, respectively }\left(\mathrm{m} \mathrm{s}^{-1}\right)\end{array}$ \\
\hline$U_{\max }, U_{\min }$ & $\begin{array}{l}\text { Maximum and minimum of the mean vertical velocity } \\
\text { profile, respectively }\left(\mathrm{m} \mathrm{s}^{-1}\right)\end{array}$ \\
\hline$<u^{\prime} v^{\prime}>_{\max }$ & Maximum value of the Reynolds stress at $x\left(\mathrm{~m}^{2} \mathrm{~s}^{-2}\right)$ \\
\hline$x$ & Distance from the inlet in the ascendant direction $(\mathrm{m})$ \\
\hline$X$ & Order of magnitude of the distance from the inlet (m) \\
\hline$y$ & Distance from the left wall $(\mathrm{m})$ \\
\hline$y_{S L}$ & $\begin{array}{l}\text { Location of the right boundary of the shear layer at } x \text {, } \\
\text { Eq.33 }(\mathrm{m})\end{array}$ \\
\hline$y_{i}$ & Right boundary location of Zone $i$ at $x$, Table 1 \\
\hline$z$ & Distance from the front lateral wall $(\mathrm{m})$ \\
\hline$\alpha(x)$ & Part of heat rate extracted from Zone V, Eq. 8 \\
\hline$\alpha^{\prime}$ & Coefficient of the order of unity, Eq. 9 \\
\hline$\beta$ & Isobaric thermal expansion of water $\left(\mathrm{K}^{-1}\right)$ \\
\hline$\Gamma=\frac{H}{b}$ & Aspect ratio \\
\hline$\delta_{i}$ & Order of magnitude of the thickness of Zone $\mathrm{i}$ at $x,(\mathrm{~m})$ \\
\hline$\Delta T(x, y)$ & $\begin{array}{l}\text { Mean temperature difference with the entry of the } \\
\text { channel }(K)\end{array}$ \\
\hline$\theta^{\prime}$ & Instantaneous temperature fluctuations $(\mathrm{K})$ \\
\hline$\theta^{*}$ & $\begin{array}{l}\text { Order of magnitude of the temperature fluctuations } \\
(\mathrm{K})\end{array}$ \\
\hline$\kappa$ & Thermal diffusivity of water $\left(\mathrm{m}^{2} \mathrm{~s}^{-1}\right)$ \\
\hline$\lambda$ & Thermal conductivity of water $\left(\mathrm{W} \mathrm{m}^{-1} \mathrm{~K}^{-1}\right)$ \\
\hline$\nu$ & Cinematic viscosity of water $\left(\mathrm{m}^{2} \mathrm{~s}^{-1}\right)$ \\
\hline$\rho$ & $\begin{array}{l}\text { Density of water at the reference temperature, }(\mathrm{kg} \\
\mathrm{m}^{-3} \text { ) }\end{array}$ \\
\hline
\end{tabular}




\begin{tabular}{|ll|}
\hline$\tilde{\rho}$ & Density of water, $\left(\mathrm{kg} \mathrm{m}^{-3}\right)$ \\
$<>$ & Time average operator \\
subscripts & \\
$c$ & Referring to the centre of the channel \\
$i$ & Order of magnitude of a quantity in Zone $i \in$ \\
& $\{I, I I \ldots V I I\}$ \\
inlet & Referring to channel inlet \\
surr & Referring to the surrounding of the channel \\
$t$ & Referring to the height of the transition \\
& \\
\hline
\end{tabular}

\section{Equations of conservation}

A description of the vertical channel is given in the companion paper and main features are recalled here. This study deals with a vertical channel with two heated plates in an infinite and isothermal domain. $x$ is the upward vertical. The height, width and depth of the channel are $H, b$ and $l$ in the $x, y$ and $z$ directions, respectively. The vertical walls in the $(x, z)$ plane are symmetrically heated with a constant heat flux $q_{w}$, the lateral walls are adiabatic. $U, V, W$ are the time-averaged velocity components in the $x, y$ and $z$ directions, respectively. $u^{\prime}, v^{\prime}, w^{\prime}$ are the corresponding instantaneous fluctuations. $\Delta T$ is the temperature difference with the temperature at the entry of the channel and $\theta^{\prime}$ is the corresponding instantaneous fluctuation.

The conservation equations that drive the flow are simplified by considering the large aspect ratio of the channel ( $H$ and $l \gg b)$. More precisely, the domain is divided into several zones (see Sec. 3), the thickness of which are much smaller than their $x$ and $z$ extensions. In the zone $i, U_{i}, V_{i}, W_{i}$ are the orders of magnitude of the velocity components along $x, y, z$ and $\Delta T_{i}$ is the order of magnitude of the departure of the temperature from the inlet temperature. In the same way, $u_{i}^{*}, v_{i}^{*}, w_{i}^{*}$ and $\theta_{i}^{*}$ are the order of magnitude of the corresponding fluctuations in the layer $i$. The correlation coefficient $c_{u v} \in[0,1]$ is defined by $\left\langle u^{\prime} v^{\prime}\right\rangle=c_{u v}\left(\left\langle u^{\prime 2}\right\rangle\left\langle v^{\prime 2}\right\rangle\right)^{1 / 2}$, where $\langle$.$\rangle is the time-$ average operator. Denoting $c_{u v, i}$ the order of magnitude of $c_{u v}$ in the layer $i$, one obtains the order of magnitude of $\left\langle u^{\prime} v^{\prime}\right\rangle$ that holds in this layer,

$$
\left\langle u^{\prime} v^{\prime}\right\rangle \simeq c_{u v, i} u_{i}^{*} v_{i}^{*}
$$


In the same way, we define $c_{u w, i}, c_{v w, i}, c_{u \theta, i}, c_{v \theta, i}, c_{w \theta, i} \in[0,1]$, the scale of the correlation coefficients corresponding to $\left\langle u^{\prime} w^{\prime}\right\rangle,\left\langle v^{\prime} w^{\prime}\right\rangle,\left\langle u^{\prime} \theta^{\prime}\right\rangle,\left\langle v^{\prime} \theta^{\prime}\right\rangle,\left\langle w^{\prime} \theta^{\prime}\right\rangle$ in the layer $i$, respectively. Due to the aspect ratio of the channel, we suppose that quantities evolve slowly along the $x$ and $z$ directions: $\frac{\partial}{\partial x} \simeq \frac{1}{X}, \frac{\partial}{\partial z} \simeq \frac{1}{l}$, where $X$ is the order of magnitude of the distance to the entrance. Concerning the scale of the derivative with respect to $y$, it depends on the layer where the derivative is considered. Thus in the layer $i$ having a thickness $\delta_{i}$, the scale of the derivative is $\frac{\partial}{\partial y} \simeq \frac{1}{\delta_{i}}$.

Our analysis is based on two main hypothesis. Firstly, the mean flow is roughly two dimensional implying that $W_{i} \ll U_{i}$ and $V_{i}$ in each layer $i$, and we consider $X \simeq l$. Secondly, due to the aspect ratio of the channel, the thickness of each layer along $y$ is small with respect to the other dimensions: $\delta_{i} \ll X$ and $l$. As a consequence, the flow is almost parallel and the equations of motion under the Oberbeck-Boussinesq approximation are reduced to their leading terms:

$$
\begin{aligned}
& \underbrace{\frac{\partial U}{\partial x}}_{\frac{U_{i}}{X}}+\underbrace{\frac{\partial V}{\partial y}}_{\frac{V_{i}}{\delta_{i}}}=0 \\
& \underbrace{U \frac{\partial U}{\partial x}}_{\frac{U_{i}^{2}}{X}}+\underbrace{V \frac{\partial U}{\partial y}}_{\frac{U_{i}^{2}}{X}}=\underbrace{-\frac{1}{\rho} \frac{\partial P_{g}}{\partial x}}_{\frac{P_{i}}{\rho X}}+\underbrace{\nu \frac{\partial^{2} U}{\partial y^{2}}}_{\frac{\nu U_{i}}{\delta_{i}^{2}}}-\underbrace{\frac{\partial\left\langle u^{\prime 2}\right\rangle}{\partial x}}_{\frac{u_{i}^{*}}{X}} \underbrace{-\frac{\partial\left\langle u^{\prime} v^{\prime}\right\rangle}{\partial y}}_{c_{u v, i} \frac{u_{i}^{*} v_{i}^{*}}{\delta_{i}}}+\underbrace{\frac{\beta g \Delta T}{\partial g}}_{\beta g \Delta T_{i}} \\
& \underbrace{U \frac{\partial V}{\partial x}}_{\frac{\delta_{i}}{X} U_{i}^{2}}+\underbrace{V \frac{\partial V}{\partial y}}_{\frac{\delta_{i}}{X} \frac{U_{i}^{2}}{X}}=\underbrace{-\frac{1}{\rho} \frac{\partial P_{g}}{\partial y}}_{\frac{P_{i}}{\rho \delta_{i}}}+\underbrace{\nu \frac{\partial^{2} V}{\partial y^{2}}}_{\frac{\nu U_{i}}{X \delta_{i}}}-\underbrace{\frac{\partial\left\langle v^{\prime 2}\right\rangle}{\partial y}}_{\frac{v_{i}^{*}}{\delta_{i}}} \\
& 0=\underbrace{-\frac{1}{\rho} \frac{\partial P_{g}}{\partial z}}_{\frac{P_{i}}{\rho X}}-\underbrace{\frac{\partial\left\langle w^{\prime 2}\right\rangle}{\partial z}}_{\frac{w_{i}^{* 2}}{X}} \\
& \underbrace{U \frac{\partial T}{\partial x}}_{\frac{U_{i} \Delta T_{i}}{X}}+\underbrace{V \frac{\partial T}{\partial y}}_{\frac{U_{i} \Delta T_{i}}{X}}=\underbrace{\kappa \frac{\partial^{2} T}{\partial y^{2}}}_{\frac{\kappa \Delta T_{i}}{\delta_{i}^{2}}}-\underbrace{\frac{\partial\left\langle u^{\prime} \theta^{\prime}\right\rangle}{\partial x}}_{c_{u \theta, i} \frac{u_{i}^{*} \theta_{i}^{*}}{\delta_{i}}}-\underbrace{\frac{\partial\left\langle v^{\prime} \theta^{\prime}\right\rangle}{\partial y}}_{c_{v \theta, i} \frac{v_{i}^{*} \theta_{i}^{*}}{\delta_{i}}} \\
& \frac{\partial T}{\partial y}=-\frac{q_{w}}{\lambda} \quad \forall x, y=0
\end{aligned}
$$




$$
\begin{aligned}
\frac{\partial T}{\partial y} & =\frac{q_{w}}{\lambda} \quad \forall x, y=b \\
U & =V=u^{\prime}=v^{\prime}=w^{\prime}=0 \quad \forall x, y=0 \text { or } b
\end{aligned}
$$

where the expression under the braces are the order of magnitude of each term in a layer $i . \quad \nu, \kappa, \beta$ and $\lambda$ are the kinematic viscosity, the thermal diffusivity, the isobaric expansion coefficient and the thermal conductivity

of the fluid. $g$ is the gravity acceleration, $\Delta T=T-T_{\text {inlet }}$ is the difference between the temperature of the fluid $T$ and the temperature at the inlet $T_{\text {inlet. }}$. Using the classical Oberbeck-Boussinesq approximation, the variation of the density with temperature is given by $\tilde{\rho}(T)=\rho\left(1-\beta\left(T-T_{\text {ref }}\right)\right.$, where $T_{\text {ref }}$ is a reference temperature and $\rho$ is the density at this reference temperature. $P_{g}=P+\rho_{\text {inlet }} g x$ is the mean driving pressure, $\rho_{\text {inlet }}$ is the density at the inlet temperature. $P_{g, i}$ is the order or magnitude of $P_{g}$ in zone i. The crosscorrelations $\left\langle u^{\prime} w\right\rangle$ and $\left\langle v^{\prime} w\right\rangle$ are supposed to be negligible.

\section{Partition of the domain}

The partition of the domain described in this section will be justified all along the article and the main ideas are given here. A schematic diagram of the zones is given in Figure 1 and the designation and notations are given in Table 1. A transition from a laminar heat transfer to a turbulent one is observed in the companion paper at $x_{t}=0.71 H$. Therefore, the first partition is defined by $x=x_{t}$, the location of the transition which defines the bottom $\left(x \leq x_{t}\right)$ and the top $\left(x \geq x_{t}\right)$ parts of the channel. As it is observed in the experimental study, heat transfer is laminar in the bottom part and it becomes turbulent in the top part. In the bottom part, Zone I is the near-wall region where the wall temperature gradient is set by the isoflux condition. At the center of the channel, Zone IV is the region where the velocity profile is flat. Considering a half-channel $(0 \leq y \leq b / 2)$, a shear layer develops between the Zones I and IV. Indeed, as it is described in the experimental study, the heating of Zone I leads to the acceleration of the fluid along the heated wall and the velocity at the center decreases due to mass conservation. A key point of this study is to split the shear layer zone according to the sign of the vertical velocity gradient $\frac{\partial U}{\partial x}$. In Zone II, the fluid accelerates along the channel whereas it decreases in Zone III. Buoyancy is found to be the driving force in Zone II and it is negligible in Zone III. The boundary between these two zones, corresponds also to the maximum of the 


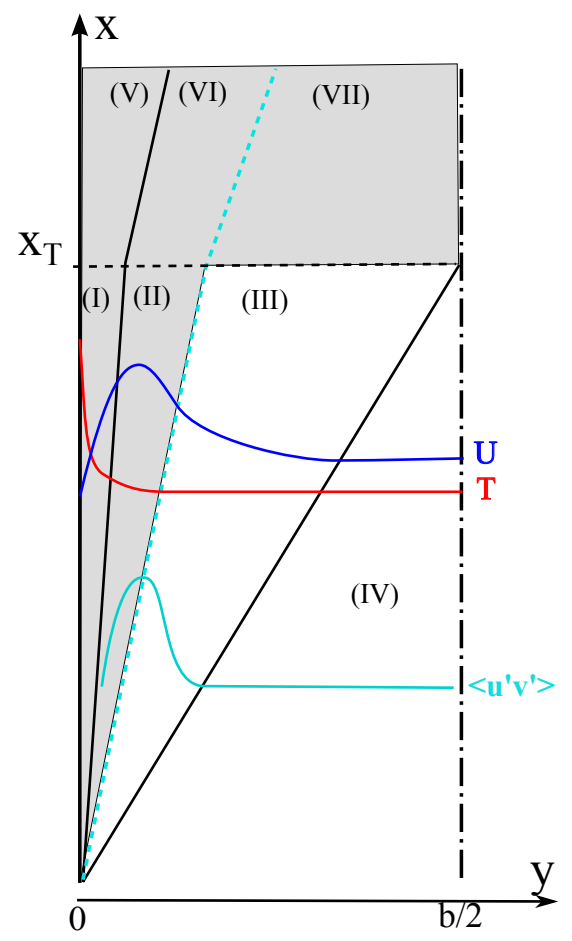

Figure 1: Partition of the half channel. The designation of the zones and their thickness are given in Table 1

Reynolds stress. In the top part, the near-wall region (Zone V) is also defined by the thermal boundary condition. Outside this zone, heat is transferred by the fluctuations to the center of the channel ([1]). Like in the bottom part, the sign of the vertical velocity gradient $\frac{\partial U}{\partial x}$ is used to define the boundary between Zones VI and VII.

\section{Results}

\subsection{Heat transfer in the near-wall region}

\subsubsection{Heat transfer below transition}

Close to the wall, molecular viscosity dominates and heat transfer is supposed to be laminar. As buoyancy is the driving force, the x-momentum equation (Eq. 2b) reduces to the balance between the viscous term and the buoyant one, and in the energy equation (Eq. 2e), the transport by fluctuations can be neglected. Moreover, as the thermal boundary condition (Eq. $2 \mathrm{f}$ or $2 \mathrm{~g}$ ) is set by the wall temperature gradient, the thickness $\delta_{I}$ of Zone I 


\begin{tabular}{|l|l|l|l|}
\hline Label & Designation of the zone & Thickness & $\begin{array}{l}\text { Right boundary } \\
\text { (notation in [1] })\end{array}$ \\
\hline$I$ & $\begin{array}{l}\text { Thermal boundary sublayer in } \\
\text { the bottom part }\end{array}$ & $\delta_{I}$ & $\begin{array}{l}y_{I}=\delta_{I} \\
\left(\delta_{T, 0}\right)\end{array}$ \\
\hline$I I$ & $\begin{array}{l}\text { Increasing vertical velocity } \\
\text { zone in the bottom part }\end{array}$ & $\delta_{I I}$ & $\begin{array}{l}y_{I I}=\delta_{I}+\delta_{I I} \\
\left(y_{u v}\right)\end{array}$ \\
\hline$I I I$ & $\begin{array}{l}\text { Decreasing vertical velocity } \\
\text { zone in the bottom part }\end{array}$ & $\delta_{I I I}$ & $\begin{array}{l}y_{I I I}=\delta_{I}+\delta_{I I}+\delta_{I I I} \\
\left(y_{S L}\right)\end{array}$ \\
\hline$I V$ & $\begin{array}{l}\text { Flat vertical velocity profile } \\
\text { zone in the bottom part }\end{array}$ & $\delta_{I V}$ & $y_{I V}=b / 2$ \\
\hline$V$ & $\begin{array}{l}\text { Thermal boundary sublayer in } \\
\text { the top part }\end{array}$ & $\delta_{V}$ & $\begin{array}{l}y_{V}=\delta_{V} \\
\left(\delta_{T, 0}\right)\end{array}$ \\
\hline$V I$ & $\begin{array}{l}\text { Decreasing vertical velocity } \\
\text { zone in the top part }\end{array}$ & $\delta_{V I}$ & $\begin{array}{l}y_{V I}=\delta_{V}+\delta_{V I} \\
\left(y_{u v}\right)\end{array}$ \\
\hline $\begin{array}{l}\text { zoncreasing vertical velocity } \\
\text { zone in the top part }\end{array}$ & $\delta_{V I I}$ & $\begin{array}{l}y_{V I I}=b / 2 \\
\left(y_{S L}\right)\end{array}$ \\
\hline
\end{tabular}

Table 1: Zones in the left half-channel $0 \leq y \leq 0.5 b$ sketched in Figure 1. Experimental values of the right boundaries are shown in the companion paper (Fig. 5).

can be defined by the ratio of the wall temperature to the wall temperature gradient. Let $U_{I}$ and $\Delta T_{I}$ denote the velocity and the temperature scales in this zone, ones obtains:

$$
\begin{aligned}
\frac{\nu U_{I}}{\delta_{I}^{2}} & \simeq \beta g \Delta T_{I} \\
\frac{\kappa X}{U_{I} \delta_{I}^{2}} & \simeq 1 \\
\frac{\Delta T_{I}}{\delta_{I}} & \simeq \frac{q_{w}}{\lambda}
\end{aligned}
$$

This leads to the classical order of magnitude of thermal boundary layer thickness along a vertical plate with isoflux condition [3]:

$$
\begin{aligned}
\delta_{I} & \simeq H\left(\frac{g \beta q_{w} H^{4}}{\lambda \nu \kappa}\right)^{-1 / 5}\left(\frac{X}{H}\right)^{1 / 5} \\
U_{I} & \simeq \frac{\kappa}{H}\left(\frac{g \beta q_{w} H^{4}}{\lambda \nu \kappa}\right)^{2 / 5}\left(\frac{X}{H}\right)^{3 / 5}
\end{aligned}
$$




$$
\Delta T_{I} \simeq \frac{q_{w} H}{\lambda}\left(\frac{g \beta q_{w} H^{4}}{\lambda \nu \kappa}\right)^{-1 / 5}\left(\frac{X}{H}\right)^{1 / 5}
$$

This result is consistent to the one obtain by Khanal and Lei [6] for the distinct thermal boundary layers regime in the steady state which is also the case for the thermal boundary layers along the left and right walls in the present study. $\delta_{I}$ is the order of magnitude of the thermal boundary sublayer (TBsL) thickness $\delta_{T, 0}$ defined in the companion paper by the following equation:

$$
\delta_{T, 0}=\frac{\lambda \Delta T_{w}}{q_{w}}
$$

In this study, we refer to Zone I as the TBsL where the temperature scale $\Delta T_{I}$ is given by the wall temperature: $\Delta T_{w}\left(x \leq x_{t}\right)=T_{w}\left(x \leq x_{t}\right)-T_{\text {inlet }} \simeq \Delta T_{I}$. Figure 2 shows the wall temperature given in the experimental study and

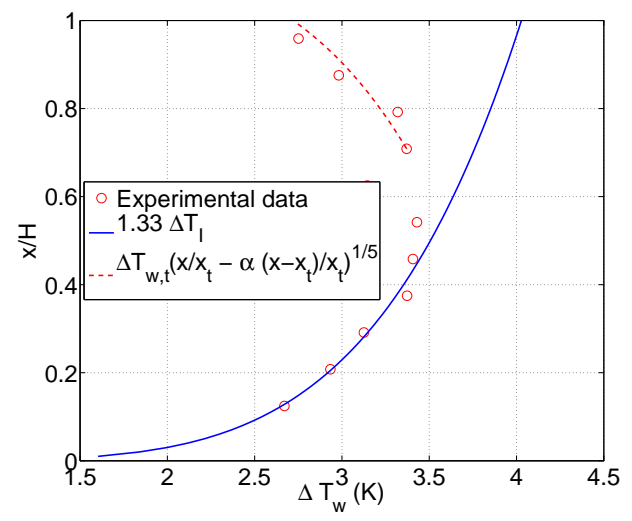

Figure 2: Experimental wall temperature (o), analytical model in the bottom part 1.33 $\Delta T_{I}$ see Eq. 4c (solid line), analytical model in the top part Eq. 12a (dash line) with $\alpha=2.6$.

$1.33 \Delta T_{I}$ where $\Delta T_{I}$ is given by Equation 4c. By taking into account for an errorbar of $\pm 0.1^{\circ} \mathrm{C}$ experimental data are well approximated by $1.33 \Delta T_{I}$. As $\delta_{I}$ is proportional to $\Delta T_{I}$, the comparison of $\delta_{T, 0}$ to $\delta_{I}$ is similar to the one shown on Figure 2 for the wall temperature. Concerning the velocity, experimental profiles (see Figure 2a in the companion paper) are not refined enough near the wall to allow an experimental determination of $U_{I}$. 


\subsubsection{Heat transfer above transition}

Above the transition, Zone $\mathrm{V}$ is the near-wall region where viscosity dominates and the wall temperature gradient is set by the isoflux condition, therefore one obtains similar expression than those given for Zone I:

$$
\begin{aligned}
\frac{\nu U_{V}}{\delta_{V}^{2}} & =\beta g \Delta T_{V} \\
\frac{\Delta T_{V}}{\delta_{V}} & =\frac{q_{w}}{\lambda}
\end{aligned}
$$

where $U_{V}, \Delta T_{V}$ are the velocity and temperature scales in Zone $\mathrm{V}$ with $\Delta T_{w}\left(x \geq x_{t}\right) \simeq \Delta T_{V}$. These equations can be written as follow:

$$
\begin{aligned}
U_{V} & =\frac{\kappa}{H} \frac{\lambda^{2} \beta g H \Delta T_{w}^{3}}{\nu \kappa q_{w}^{2}} \\
\delta_{V} & =H \frac{\lambda \Delta T_{w}}{q_{w} H}
\end{aligned}
$$

But, unlike Zone I, heat from the wall is no longer completely lifted upward but a significant part is transported through Zone $\mathrm{V}$ by molecular diffusion and then convected to the center of the channel. Let $\alpha(x) q_{w}$ denotes the part of heat rate extracted from Zone $\mathrm{V}$ by the mean flow and the fluctuations:

$$
\left[V \Delta T+\left\langle v^{\prime} \theta^{\prime}\right\rangle\right]_{x, \delta_{V}} \simeq \alpha \frac{q_{w}}{\rho C_{p}}
$$

To obtain the wall temperature in this zone an energy budget is done in the near-wall region shown in Figure 3. Let $Q_{\text {conv }, V}$ and $Q_{\text {conv }, V}^{\prime}$ denote the vertical heat rate through Zone $\mathrm{V}$ and the horizontal heat rate at the boundary V-VI, respectively.

$$
\begin{aligned}
q_{w} x & =Q_{\mathrm{conv}, V}+Q_{\mathrm{conv}, V}^{\prime} \\
& =\rho C_{p} \int_{0}^{\delta_{V}} U \Delta T d y+\rho C_{p} \int_{x_{t}}^{x}\left[V \Delta T+\left\langle v^{\prime} \theta^{\prime}\right\rangle\right]_{x, \delta_{V}} d x \\
& \simeq \alpha^{\prime} \rho C_{p} U_{V} \Delta T_{V} \delta_{V}+\rho C_{p}\left(x-x_{t}\right)\left[V \Delta T+\left\langle v^{\prime} \theta^{\prime}\right\rangle\right]_{x, \delta_{V}}
\end{aligned}
$$

where $\alpha^{\prime}=\left(U_{V} \Delta T_{w} \delta_{V}\right)^{-1} \int_{0}^{\delta_{V}} U \Delta T d y$ is supposed to be independent on $x$, meaning that the solution $(U, \Delta T)$ in Zone $\mathrm{V}$ is self-similar: $U=U_{V} f\left(\frac{y}{\delta_{V}}\right)$, $\Delta T=\Delta T_{V} h\left(\frac{y}{\delta_{V}}\right)$. 


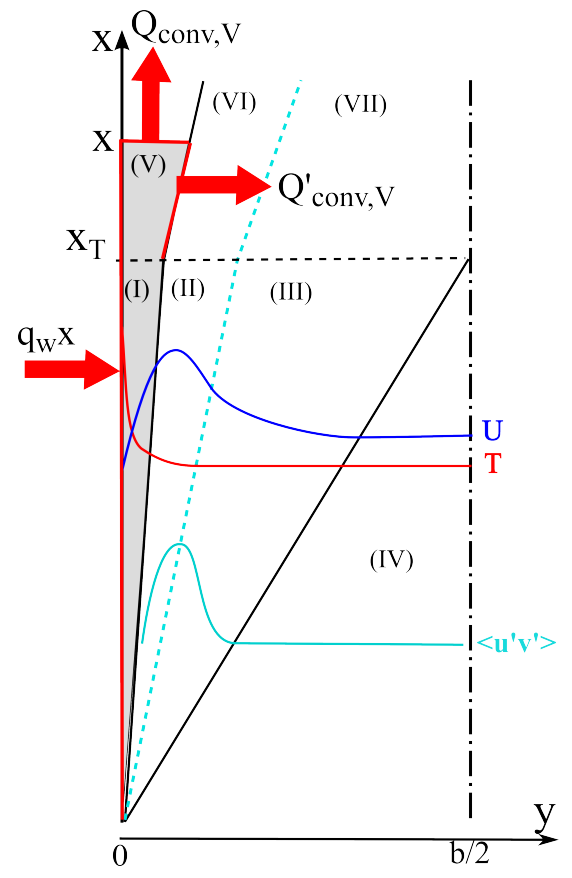

Figure 3: Energy budget in the near wall region.

Equations 8 and 9 lead to:

$$
U_{V} \Delta T_{V} \delta_{V} \simeq \frac{q_{w} x_{t}}{\alpha^{\prime} \rho C_{p}}\left[\alpha+(1-\alpha) \frac{x}{x_{t}}\right]
$$

Using Equations 7 and 10, one obtains the temperature, velocity and thickness scales in Zone V:

$$
\begin{aligned}
\Delta T_{V} & =\frac{q_{w} x_{t}}{\lambda}\left(\frac{\beta g q_{w} x_{t}^{4}}{\lambda \nu \kappa}\right)^{-1 / 5}\left(\frac{\alpha}{\alpha^{\prime}}+\frac{1-\alpha}{\alpha^{\prime}} \frac{x}{x_{t}}\right)^{1 / 5} \\
\delta_{V} & =x_{t}\left(\frac{\beta g q_{w} x_{t}^{4}}{\lambda \nu \kappa}\right)^{-1 / 5}\left(\frac{\alpha}{\alpha^{\prime}}+\frac{1-\alpha}{\alpha^{\prime}} \frac{x}{x_{t}}\right)^{1 / 5} \\
U_{V} & =\frac{\kappa}{x_{t}}\left(\frac{\beta g q_{w} x_{t}^{4}}{\lambda \nu \kappa}\right)^{2 / 5}\left(\frac{\alpha}{\alpha^{\prime}}+\frac{1-\alpha}{\alpha^{\prime}} \frac{x}{x_{t}}\right)^{3 / 5}
\end{aligned}
$$

These equations can be rewritten in terms of $\Delta T_{I, t}, \delta_{I, t}$ and $U_{I, t}$ which are 
the orders of magnitude at $x=x_{t}$ found in Zone I (Eqs. 4):

$$
\begin{aligned}
\Delta T_{V} & =\alpha^{\prime-1 / 5} \Delta T_{I, t}\left(\alpha+(1-\alpha) \frac{x}{x_{t}}\right)^{1 / 5} \\
\delta_{V} & =\alpha^{\prime-1 / 5} \delta_{I, t}\left(\alpha+(1-\alpha) \frac{x}{x_{t}}\right)^{1 / 5} \\
U_{V} & =\alpha^{\prime-3 / 5} U_{I, t}\left(\alpha+(1-\alpha) \frac{x}{x_{t}}\right)^{3 / 5} .
\end{aligned}
$$

As the wall temperature in the top part is clearly a decreasing function of $x$ (Fig. 2), Equation 11a implies $\alpha \geq 1$ which means that the local heat rate at the boundary between Zones V and VI exceeds the wall heat rate (Eq. 8).

At the position of the transition,

$$
\begin{aligned}
\Delta T_{V, t} & =\alpha^{-1 / 5} \Delta T_{I, t} \\
\delta_{V, t} & =\alpha^{\prime-1 / 5} \delta_{I, t} \\
U_{V, t} & =\alpha^{\prime-3 / 5} U_{I, t}
\end{aligned}
$$

$\Delta T_{V, t}$ is chosen equal to the experimental value of the wall temperature at the transition:

$$
\Delta T_{V, t}=\Delta T_{w, t}
$$

Equation $4 \mathrm{c}$ at $x=x_{t}$, Equations $12 \mathrm{a}$ and 13a give the value of $\alpha^{\prime}$ :

$$
\alpha^{\prime}=\left(\frac{\beta g q_{w} H^{4}}{\lambda \nu \kappa}\right)^{-1}\left(\frac{q_{w} H}{\lambda \Delta T_{w, t}}\right)^{5} \frac{x_{t}}{H} \approx 0.4
$$

This value of $\alpha^{\prime}$ which comes from experimental results in the companion paper, is consistent with the following self-similar solution in Zone $\mathrm{V}: U=$ $U_{V}(x)\left(\frac{y}{\delta_{V}}\right)^{0.2}$ and $\Delta T=\Delta T_{w}(x)\left(1-\frac{y}{\delta_{V}}\right)$. However, these solutions come from scale analysis and should not be taken as exact functions, but they allow to check the consistency of the results.

Moreover, as the factors $\alpha^{\prime-3 / 5}=1.7$ and $\alpha^{\prime-1 / 5}=1.2$ are of the order of the unity, one may consider that the continuity of the scales is ensured between Zones I and $\mathrm{V}: U_{I, t} \simeq U_{V, t}, \delta_{I, t} \simeq \delta_{V, t}, \Delta T_{I, t} \simeq \Delta T_{V, t}$. Figure 2 shows the comparison between the experimental results and Equation $12 \mathrm{a}$ with $\alpha=2.6$. 
In fact, the extension of the top part of the channel is not long enough to obtain accurate values of these coefficients. However, with a constant coefficient $\alpha=2.6$, the expression given in Equation 12a is consistent with the experimental data as shown in Figure 2. Experimental data with a longer top part of the channel is required to find a more accurate expression of the function $\alpha(x)$.

\subsection{Pressure field in the channel}

\subsubsection{Velocity and pressure field in Zone IV}

The experimental study shows a flat velocity profile at the entry. Let $U_{\text {inlet }}$ denotes the velocity at the entry. Zone IV is defined as the region at the center of the channel, where the vertical velocity profile remains flat. Indeed, as shown in the companion paper, the velocity profile is flat at the entry of the channel and a positive momentum is transported from the peak of the velocity profile toward the center of the channel as the fluid rises. Therefore, Zone IV is the central region that is not directly influenced by this momentum transport. The corresponding velocity field is:

$$
\begin{aligned}
& U_{I V}=U_{\min }(x) \\
& V_{I V}=\left(\frac{b}{2}-y\right) \frac{\partial U_{\min }}{\partial x}
\end{aligned}
$$

where $U_{\min }(x)$ is the vertical velocity at the center of the channel and the expression of $V_{I V}$ results from the mass conservation. By using Equations $2 \mathrm{~b}$ and $2 \mathrm{c}$, one can find the following expression for the velocity and pressure in Zone IV (see Appendix A for details):

$$
\begin{aligned}
U_{I V} & =U_{\min }(x)=-A_{m} x+U_{\text {inlet }} \\
V_{I V} & =-A_{m}\left(\frac{b}{2}-y\right) \\
\frac{P_{g I V}}{\rho}+\left\langle u^{\prime 2}\right\rangle & =\left(\frac{P_{g I V}}{\rho}+\left\langle u^{\prime 2}\right\rangle\right)(x=0, y)+\frac{U_{\text {inlet }}^{2}-U_{\text {min }}^{2}}{2}
\end{aligned}
$$

Figure 4 shows the velocity at the centre of the channel and the best linear fit of the curve. The difference between the experimental data and the linear fit is less than $9 \%$ below the transition exceeding the uncertainty for velocity measurement $\pm 4 \%$ (see [1]). However, as it will be mentioned at the end of Section 4.2 .2 , the hypothesis $\frac{\partial\left(\left\langle u^{\prime 2}\right\rangle-\left\langle v^{\prime 2}\right\rangle\right)}{\partial y}=0$ in the entire Zone IV is 


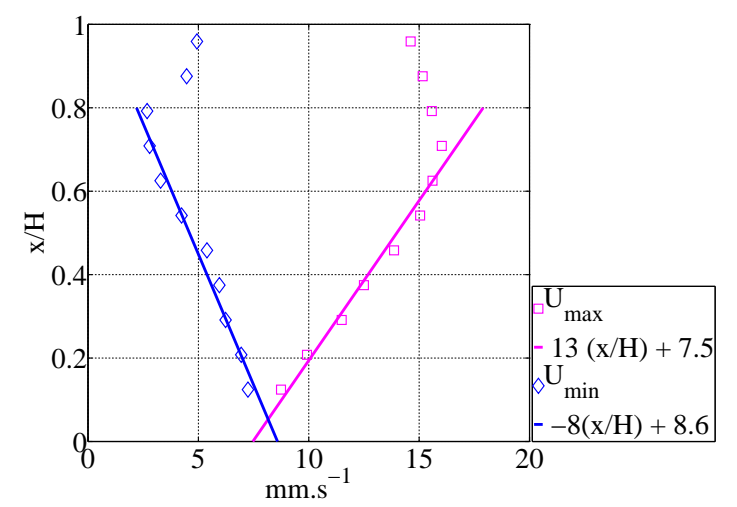

Figure 4: Maximum velocity and velocity at the centre of the channel and the corresponding linear best fits (values are expressed in $\mathrm{mm} \cdot \mathrm{s}^{-1}$ )

probably too strong which could explain this large difference between experimental data and Equation 17a. The linear approximation leads to an inlet velocity of $8.6 \mathrm{~mm} \cdot \mathrm{s}^{-1}$. As the flow is quasi-parallel, it is possible to extend the validity domain of Equation 17c to the whole section in the bottom part of the channel except in the near-wall region as shown in the next section.

\subsubsection{Expression of the pressure below transition}

We evaluate the order of magnitude of the terms of Equation 2c from the data given in the experimental study where the aspect ratio is $\Gamma=H / b=$ 10.4 and the turbulent intensity is around $I_{u, \text { entry }}=35 \%$ at the entry of the channel. Thus, the ratio between the derivative of the rms velocity $\left(\frac{\partial\left\langle v^{2}\right\rangle}{\partial y}\right)$ and the convective term leads to the following order of magnitude:

$$
\frac{v_{i}^{* 2}}{U_{i}^{2}} \frac{X^{2}}{\delta_{i}^{2}} \simeq I_{u, \text { entry }} \Gamma^{2} \frac{b^{2}}{\delta_{i}^{2}} \simeq 35 \frac{b^{2}}{\delta_{i}^{2}} \gg 1 .
$$

Therefore, the convective terms are negligible with respect to the turbulent momentum diffusion one. Moreover, in Equation 2c, the viscous term will appear not to dominate in Zones II, III and IV (see Sec. 4.3.2, 4.3.3). As a consequence, the convective terms and the viscous terms can be neglected in the y-momentum equation and Equation 2c reduces to:

$$
0=-\frac{1}{\rho} \frac{\partial P_{g}}{\partial y}-\frac{\partial\left\langle v^{2}\right\rangle}{\partial y}
$$




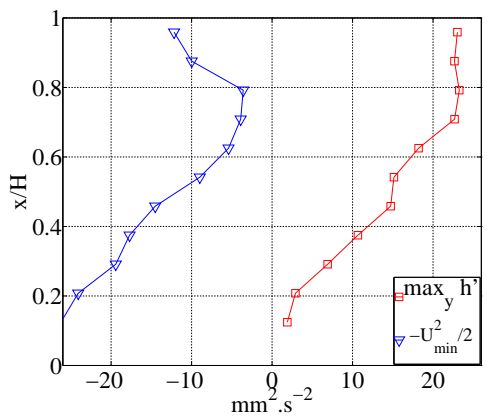

Figure 5: $-\frac{1}{2} U_{\min }^{2}$ and $\max _{y} h^{\prime}$ along the channel.

Equations $2 \mathrm{~d}$ and 18 show that the pressure differences in the spanwise directions are balanced by velocity fluctuations.

To go further, some hypotheses are required. As the geometry and the boundary conditions are symmetrical with respect to the plane $z=0.5 l$, we suppose that the rms values of the velocity fluctuations are uniform in the z-direction. Therefore, Equation 2d leads to a zero pressure gradient in the z-direction. Then by using Equations 17c and 18, one obtains (see Appendix B for details):

$$
\begin{aligned}
\frac{\partial}{\partial x}\left[\frac{P_{g}}{\rho}+\left\langle u^{\prime 2}\right\rangle\right] & =-\underbrace{U_{\min } \frac{\partial U_{\min }}{\partial x}}_{\frac{\bar{U}^{2}}{X}} \\
& +\underbrace{\left[\frac{\partial\left\langle u^{\prime 2}\right\rangle}{\partial x}-\frac{\partial\left\langle v^{\prime 2}\right\rangle}{\partial x}\right](x, y)-\left[\frac{\partial\left\langle u^{\prime 2}\right\rangle}{\partial x}-\frac{\left.\partial\left\langle v^{\prime 2}\right\rangle\right]}{\partial x}\right](x, b / 2)}_{\frac{u^{*} 2}{X}}
\end{aligned}
$$

Let's denote $h^{\prime}(x, y)=\left[\left\langle u^{\prime 2}\right\rangle-\left\langle v^{\prime 2}\right\rangle\right](x, y)-\left[\left\langle u^{\prime 2}\right\rangle-\left\langle v^{\prime 2}\right\rangle\right](x, b / 2)$. Then Equation 19 can be written:

$$
\frac{\partial}{\partial x}\left[\frac{P_{g}}{\rho}+\left\langle u^{\prime 2}\right\rangle\right]=\frac{1}{2} \frac{\partial\left(-U_{\min }^{2}\right)}{\partial x}+\frac{\partial h^{\prime}(x, y)}{\partial x}
$$

Figure 5 shows experimental values of $-U_{\min }^{2} / 2$ and $\max _{y} h^{\prime}$, the maximum of $h^{\prime}$ with respect to $y$ along the channel. These two quantities evolve in a similar way below the transition $\left(x<x_{t}\right)$ suggesting that the two terms in the right-hand side of Equation 20 are of the same order of magnitude. 
Therefore, all the velocity fluctuation terms in Equation 19 can be taken into account by doubling the first term and Equation 19 can be written:

$$
\frac{\partial}{\partial x}\left[\frac{P_{g}}{\rho}+\left\langle u^{\prime 2}\right\rangle\right] \approx-2 U_{\min } \frac{\partial U_{\min }}{\partial x}
$$

This equation has to be compared with the expression of the pressure in Zone IV obtained from Equation 17c:

$$
\frac{\partial}{\partial x}\left[\frac{P_{g, I V}}{\rho}+\left\langle u^{\prime 2}\right\rangle\right]=-U_{\min } \frac{\partial U_{\min }}{\partial x}
$$

The difference is due to the assumption, $\frac{\partial\left\langle u^{\prime 2}\right\rangle}{\partial y}=\frac{\partial\left\langle v^{\prime 2}\right\rangle}{\partial y}=0$ used in the whole Zone IV (see Appendix A). Therefore, the exact value of the pressure gradient lies between these approximations and Equation 21 is an acceptable expression of the pressure gradient in the Zones II, III and IV. As a consequence, Equation $2 \mathrm{~b}$ in these zones can be written:

$$
U \frac{\partial U}{\partial x}+V \frac{\partial U}{\partial y}=2 U_{\min } \frac{\partial U_{\min }}{\partial x}+\nu \frac{\partial^{2} U}{\partial y^{2}}-\frac{\partial\left\langle u^{\prime} v^{\prime}\right\rangle}{\partial y}+\beta g \Delta T
$$

\subsubsection{Expression of the pressure above transition}

Equations $2 \mathrm{~d}$ and 18 remain valid above the transition. As in the bottom part, rms values of the velocity fluctuations are supposed to be independent on $z$, so is the mean driving pressure. Therefore, using Equation 18, the pressure in Zones VI and VII can be written:

$$
\frac{P_{g}}{\rho} \simeq f(x)-\left\langle v^{\prime 2}\right\rangle
$$

In the companion paper, the rms velocity components are shown to be weakly dependent on $x$ above the transition. Supposing that $\left\langle u^{\prime 2}\right\rangle$ and $\left\langle v^{\prime 2}\right\rangle$ are independent on $x$ leads to:

$$
\frac{\partial}{\partial x}\left(\frac{P_{g}}{\rho}+\left\langle u^{\prime 2}\right\rangle\right) \simeq \frac{\partial f}{\partial x}(x)
$$

This equation is used in Section 4.3.4.

In Section 4.3.4, an expression is given for the momentum equation above the transition (Eq. 39) that is valid in Zones VI and VII:

$$
-\frac{\partial f}{\partial x}(x)+\beta g \Delta T_{c}(x) \simeq U_{\max } \frac{\partial U_{\max }}{\partial x}+U_{\min } \frac{\partial U_{\min }}{\partial x} .
$$


In this equation, $\Delta T_{c}$ is the difference between the temperature of the bulk (outside the TBsL) and the temperature at the entry. $\Delta T_{c}$ is supposed to be independent on $y$ (see Sec. 4.3.4). Supposing that $\left\langle v^{\prime 2}\right\rangle$ is independent on $x$, the pressure in the top part of the channel is deduced from Equations 23 and 39:

$$
\frac{P_{g}}{\rho}-\frac{P_{g, t}}{\rho} \simeq+g \beta \int_{x_{t}}^{x} \Delta T_{c} d x-\frac{1}{2}\left(U_{\max }^{2}+U_{\min }^{2}\right)+\frac{1}{2}\left(U_{\max , t}^{2}+U_{\min , t}^{2}\right)
$$

Considering the expression of the pressure gradient in the bottom part (Eq. 21), one obtains the following expression for the pressure at the transition:

$$
\frac{P_{g, t}}{\rho}-\frac{P_{g, \text { inlet }}}{\rho} \simeq\left\langle u^{\prime 2}\right\rangle_{\text {inlet }}-\left\langle u^{\prime 2}\right\rangle_{t}-U_{\text {min }, t}^{2}+U_{\text {inlet }}^{2}
$$

Equations 25 and 26 lead to the pressure in the top part of the channel:

$$
\begin{aligned}
\frac{P_{g}}{\rho}+\left\langle u^{\prime 2}\right\rangle_{t} & \simeq \frac{P_{g, \text { inlet }}}{\rho}+\left\langle u^{\prime 2}\right\rangle_{\text {inlet }}+g \beta \int_{x_{t}}^{x} \Delta T_{c} d x \\
& -\frac{1}{2}\left(U_{\max }^{2}+U_{\min }^{2}\right)+\frac{1}{2}\left(U_{\max , t}^{2}-U_{\min , t}^{2}\right)+U_{\text {inlet }}^{2} \quad x \geq x_{t}
\end{aligned}
$$

To obtain a simplified expression of the pressure, the temperature at the centre is taken as constant in the upper part of the channel: $\Delta T_{c} \simeq \Delta T_{c, t} \simeq$ $0.4^{\circ} \mathrm{C}$ (Experimental data are shown in Figure 9). Moreover, from experimental data, one can show that the velocity terms in the right-hand side of Equation 27 are found to be small with respect to the temperature term, so they are reduced to their value at the transition. Finally, the pressure in the channel can be approximated by the following expressions:

$$
\begin{aligned}
\frac{P_{g}}{\rho}+\left\langle u^{\prime 2}\right\rangle & \simeq \frac{P_{g, \text { inlet }}}{\rho}+\left\langle u^{\prime 2}\right\rangle_{\text {inlet }}+U_{\text {inlet }}^{2}-U_{\text {min }}^{2} & & x \leq x_{t} \\
\frac{P_{g}}{\rho}+\left\langle u^{\prime 2}\right\rangle_{t} & \simeq \frac{P_{g, \text { inlet }}}{\rho}+\left\langle u^{\prime 2}\right\rangle_{\text {inlet }}+U_{\text {inlet }}^{2}-U_{\text {min }, t}^{2} & & \\
& +g \beta \Delta T_{c, t}\left(x-x_{t}\right) & & x \geq x_{t}
\end{aligned}
$$

Equations 28a and 28b are plotted on Figure 6. The heating of the bulk flow above the transition (Zones VI and VII) results in a large increase in the longitudinal pressure gradient. This result is discussed in Section 5. 


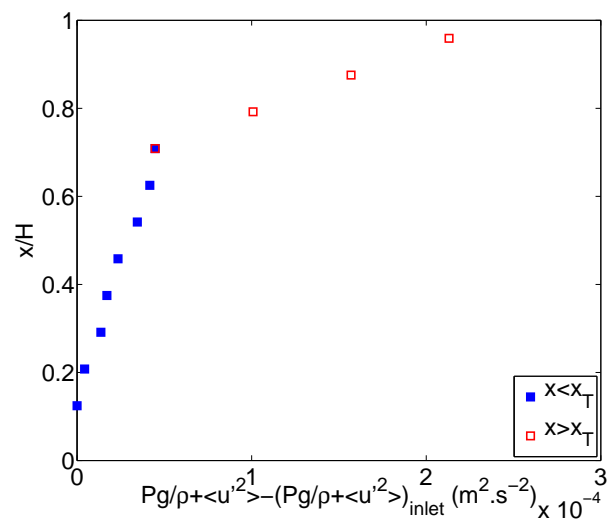

Figure 6: Pressure profile $\left(\frac{P_{g}}{\rho}+\left\langle u^{\prime 2}\right\rangle-\left[\frac{P_{g}}{\rho}+\left\langle u^{\prime 2}\right\rangle\right]_{\text {inlet }}\right)$ computed from Equations 28a and $28 \mathrm{~b}$

\subsection{Characterization of the shear layer}

\subsubsection{Definition of the boundary between Zone II and Zone III}

Vertical velocity increases along the channel in Zone I due to buoyant effect (Sections 4.1.1) whereas it decreases in Zone IV due to mass conservation (Section 4.2.1). The boundary between Zones II and III (Figure 1) is basically defined by the location where the vertical velocity is independent on $x$. This boundary $y_{I I}$ is defined by the following equation:

$$
\frac{\partial U}{\partial x}=0 \quad x, y=y_{I I}
$$

As the velocity gradient along $y$ is negative between the velocity peak and the channel center, a change of sign of the velocity gradient along $x$ on each side of this boundary tends to increase the local slope of the velocity profile which increase the fluctuations. Therefore, one may suppose that this boundary corresponds also to the maximum Reynolds stress location. Indeed, between Zones I and IV, the Reynolds stress exhibits a maximum as shown in the experimental study. The sign of the momentum diffusion term $-\frac{\partial\left\langle u^{\prime} v^{\prime}\right\rangle}{\partial y}$ in Equation $2 \mathrm{~b}$ changes on both sides of this maximum: on its left $\left(-\frac{\partial\left\langle u^{\prime} v^{\prime}\right\rangle}{\partial y}<0\right)$, a vertical layer of fluid is slowed down by the adjacent layers whereas it is accelerated on its right $\left(-\frac{\partial\left\langle u^{\prime} v^{\prime}\right\rangle}{\partial y}>0\right)$. 


\subsubsection{Accelerating zone below transition: Zone II}

In Zone II, the momentum diffusion term is negative in Equation 22. A closer look at the sign of each term of this equation shows that buoyancy is the only driving force of the flow. Among the other terms, none seems to dominate, therefore, we suppose that they are of the same order of magnitude which leads to:

$$
\frac{\partial\left\langle u^{\prime} v^{\prime}\right\rangle}{\partial y} \simeq U \frac{\partial U}{\partial x} \simeq \frac{1}{5} \beta g \Delta T
$$

Considering the following orders of magnitude $\left\langle u^{\prime} v^{\prime}\right\rangle \simeq\left\langle u^{\prime} v^{\prime}\right\rangle_{\max }, \frac{\partial U^{2}}{\partial x} \simeq$ $\frac{U_{\max }^{2}-U_{\text {inlet }}^{2}}{X}$, one obtains:

$$
\begin{aligned}
\left\langle u^{\prime} v^{\prime}\right\rangle_{\max } & \simeq \frac{U_{\text {max }}^{2}-U_{\text {inlet }}^{2}}{X} \delta_{I I} \\
\beta g \Delta T_{I I} & \simeq 5 \frac{U_{\text {max }}^{2}-U_{\text {inlet }}^{2}}{X}
\end{aligned}
$$

where $\Delta T_{I I}$ is the temperature scale in Zone II. Figure 7 shows that the experimental values of $\left\langle u^{\prime} v^{\prime}\right\rangle_{\max }$ are well approximated by the expression given in Equation 30a.

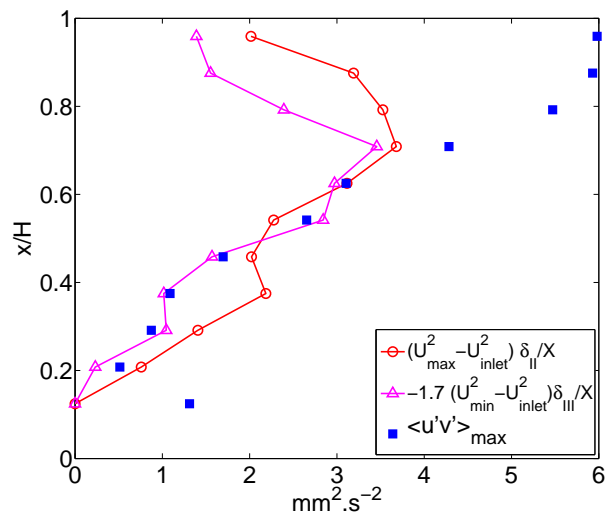

Figure 7: Reynolds stress in the bottom part: comparison of the experimental data with Equations 30a (०) and $34(\triangle)$

Concerning the energy equation (Eq. 2e), the heat transfer model in Zone I implies that the energy is contained inside the TBsL. Indeed, let 
$Q_{\text {conv }, I}$ denotes the vertical heat rate at a location $x$ in Zone I. Its order of magnitude can be calculated from Equations 4:

$$
Q_{\mathrm{conv}, I} \simeq \rho C_{p} U_{I} \Delta T_{I} \delta_{I}=q_{w} x .
$$

It corresponds to the whole energy injected at the wall below $x$. However, to be consistent with the slight heating of Zone II (Eq. 30b), the heat transfer from Zone I to Zone II is not zero. If one supposes that the convective terms are balanced by the diffusive one in Zone II, it would lead to the following order of magnitude for $\delta_{I I}: \sqrt{\frac{\kappa X}{U_{\max }}}$. But this scale is found to be much lower than the experimental data shown in the companion paper: $\sqrt{\frac{\kappa X}{U_{\max }}} \ll y_{u v}$. Therefore, $\sqrt{\frac{\kappa X}{U_{\max }}}$ is not the order of magnitude of $\delta_{I I}$ and the diffusive term is negligible in the energy equation in Zone II. As a consequence, the left-hand side of the energy equation (Eq. 2e) is balanced by a small turbulent transport at the boundary I-II. This transport by fluctuations are attributed to the relatively high turbulent intensity observed at the entry $\left(I_{u, \text { entry }}=35 \%\right)$ : the fluctuating flow entering the channel tends to extract some fluid from the TBsL to Zone II. However, we claim that this heat transported from Zone I to Zone II remains negligible with respect to the heat transported in Zone I but it induces a heating that has an impact on the momentum transport as explained at the beginning of the section. Indeed, the balance between the convective terms and the turbulent transport in Equation 2e in Zone II is written:

$$
U \frac{\partial \Delta T}{\partial x}+V \frac{\partial \Delta T}{\partial y} \simeq \frac{\partial\left\langle v^{\prime} \theta^{\prime}\right\rangle}{\partial y}
$$

which leads to the following order of magnitude:

$$
\left\langle v^{\prime} \theta^{\prime}\right\rangle X \simeq U_{\max } \Delta T_{I I} \delta_{I I}
$$

where $U_{I I}=U_{\max }$. Using Equation 30b, the experimental value of $U_{\max }$ and an approximation of $\delta_{I I}$ (see [1] Fig.5, $y_{u v}$ ), one obtains an estimate of $\left\langle v^{\prime} \theta^{\prime}\right\rangle$ in Zone IV:

$$
\left\langle v^{\prime} \theta^{\prime}\right\rangle \simeq 0.5 \frac{q-w}{\rho C_{p}}
$$

The fact that this ratio is not negligible with respect to 1 can be attributed to prefactors in the expressions of the scales in Zone II (Eqs. 30b and 32). 
Indeed, the good agreement between the experimental value and the wall temperature model (Eq. 4c and Fig. 2) suggests that the energy is mainly contained in Zone I. Moreover, a more efficient turbulent heat transfer takes place in the top part of the channel at the Boundary V-VI and the comparison of the experimental data with the heat transfer model in Zone $\mathrm{V}$ exhibits a much larger horizontal turbulent heat flux (Eq. 8 with $\alpha=2.6$ ). As a consequence, despite the existence of a weak heat transfer due to the fluctuations at the Boundary I-II, it is not a turbulent regime as it has almost no impact on the overall heat transfer in the bottom part. But the resulting slight heating of Zone II has a real impact on the vertical momentum transport as buoyancy is the driving force in this zone.

\subsubsection{Decelerating zone below transition: Zone III}

The left boundary of Zone III $\left(y_{I I}\right)$ is defined by Equation 29. Its right boundary corresponds to the edge of the shear layer which is denoted $y_{S L}$ in the companion paper. $y_{S L}$ is defined as the closest position on the right of the velocity peak where,

$$
U\left(x, y_{S L}\right)=1.2 U_{\min }
$$

Indeed, the velocity uncertainty is estimated to $\pm 4 \%$ in the experimental study, so a coefficient of 1.2 corresponds to the detection of an overspeed that it is larger than the errorbar. This definition also means that $U_{\min }$ is the relevant velocity scale in Zone III up to a multiplicative factor. In this zone, the turbulent momentum diffusion term is positive and it is supposed to be larger than the buoyant and viscous terms. Therefore, Equation 22 can be written:

$$
\left\langle u^{\prime} v^{\prime}\right\rangle_{\max } \simeq-a \frac{U_{\min }^{2}-U_{\text {inlet }}^{2}}{X} \delta_{I I I}
$$

where $a$ is a multiplicative factor of the order of the unity. This expression is plotted in Figure 7 with $a=1.7$ and it fits with the experimental data.

\subsubsection{Shear layer above transition}

Above the transition, heat is transported by the fluctuations from Zone $\mathrm{V}$ to the centre of the channel which leads to the heating of the Zones VI and VII to a temperature $T_{c}$ (see [1]). We suppose that due to the mixing, $T_{c}$ 
depends only on $x$. The boundary between Zone VI and VII is given by the location where vertical velocity is independent on $x$ :

$$
\frac{\partial U}{\partial x}=0 \quad x, y=y_{V I}
$$

As for the boundary between Zones II and III, this location is also supposed to correspond to the maximum of the Reynolds stress. In Zone VI, turbulent momentum tends to slow down the fluid whereas it tends to be accelerated in Zone VII. By taking into account Equation 24, the x-momentum equation (Equation 2b) can be written in Zones VI and VII:

$$
U \frac{\partial U}{\partial x}+V \frac{\partial U}{\partial y}=-\frac{\partial f}{\partial x}(x)+\beta g \Delta T_{c}(x)-\frac{\partial\left\langle u^{\prime} v^{\prime}\right\rangle}{\partial y}
$$

where the viscous term is neglected. This equation is differentiated with respect to $y$ :

$$
\frac{\partial}{\partial y}\left(U \frac{\partial U}{\partial x}\right)+V \frac{\partial^{2} U}{\partial y^{2}}+\frac{\partial V}{\partial y} \frac{\partial U}{\partial y}=-\frac{\partial^{2}\left\langle u^{\prime} v^{\prime}\right\rangle}{\partial y^{2}}
$$

To evaluate $\left\langle u^{\prime} v^{\prime}\right\rangle_{\max }$, we estimate the order of magnitude of each term at the boundary between Zones VI and VII. In Zones VI, the vertical velocity profile is roughly concave whereas it is convex in Zone VII (Figure 1). Therefore, one expects an inflexion point around the boundary between these two zones and the term $V \frac{\partial^{2} U}{\partial y^{2}}$ is supposed to be negligible. In the same way, the fluid is slowed down $\left(\frac{\partial U}{\partial x}<0\right)$ in almost the whole Zone VI whereas it is accelerated $\left(\frac{\partial U}{\partial x}>0\right)$ in Zone VII. Due to mass conservation, the velocity gradient $\frac{\partial V}{\partial y}$ is positive in Zone VI and negative in Zone VII. Therefore, this gradient is zero around the boundary between Zones VI and VII and the term $\frac{\partial V}{\partial y} \frac{\partial U}{\partial y}$ can be neglected. At $y=\delta_{V I}$ in the top part, the order of magnitude of Equation 37 leads to:

$$
\begin{aligned}
\left\langle u^{\prime} v^{\prime}\right\rangle_{\max } & \simeq \frac{b}{2}\left(U_{\min } \frac{\partial U_{\min }}{\partial x}-U_{\max } \frac{\partial U_{\max }}{\partial x}\right) \\
& \simeq \frac{b}{2}\left(U_{\min } \frac{U_{\min }-U_{\min , \mathrm{t}}}{x-x_{t}}-U_{\max } \frac{U_{\max }-U_{\max , \mathrm{t}}}{x-x_{t}}\right)
\end{aligned}
$$

where $b / 2$ is taken as the relevant length scale for the derivative with respect to $y, U_{V I}=U_{\max }$ and $U_{V I I}=U_{\min }$ are the velocity scales in Zones VI 


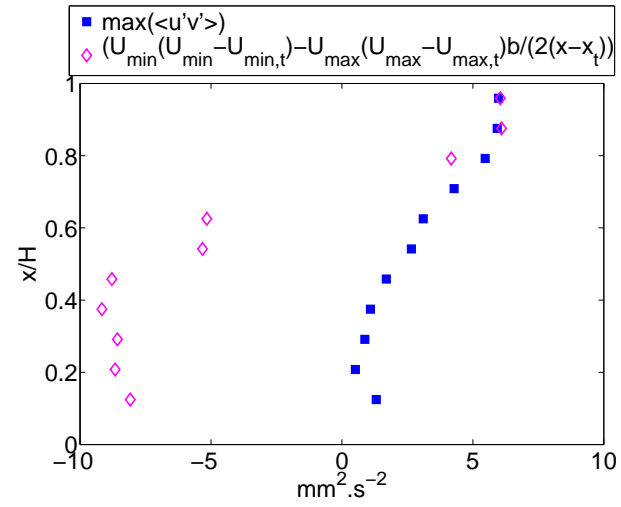

Figure 8: Maximum Reynolds stress: experimental value $(\boldsymbol{\square})$ and corresponding model $(\diamond$ Equation 38) that is valid above the transition $(x / H \geq 0.71)$.

and VII, respectively. $U_{\min , \mathrm{t}}$ and $U_{\max , \mathrm{t}}$ are the minimum and maximum vertical velocity at the transition, respectively. The comparison with the experimental data is plotted in Figure 8.

From the expression of the maximum Reynolds stress, we estimate the magnitude of the sum of pressure and buoyant terms in Equation 36. However, this estimation is tricky because it cumulates uncertainties in the estimate of the derivatives and of the order of magnitude of the Reynolds stress. Therefore, we give an estimate of the right-hand side that is consistent with the order of magnitude of the Reynolds stress.

Equation 36 leads to:

$$
\begin{aligned}
2 U_{\max } \frac{\partial U_{\max }}{\partial x} & \simeq-\frac{\partial f}{\partial x}(x)+\beta g \Delta T_{c}(x)-2 \frac{\left\langle u^{\prime} v^{\prime}\right\rangle_{\max }}{b} & & \text { Zone VI } \\
2 U_{\min } \frac{\partial U_{\min }}{\partial x} & \simeq-\frac{\partial f}{\partial x}(x)+\beta g \Delta T_{c}(x)+2 \frac{\left\langle u^{\prime} v^{\prime}\right\rangle_{\max }}{b} & & \text { Zone VII }
\end{aligned}
$$

Finally, one obtains:

$$
\begin{aligned}
-\frac{\partial f}{\partial x}(x)+\beta g \Delta T_{c}(x) & \simeq U_{\max } \frac{\partial U_{\max }}{\partial x}+U_{\min } \frac{\partial U_{\min }}{\partial x} \\
& \simeq U_{\max } \frac{U_{\max }-U_{\max , \mathrm{t}}}{X-X_{t}}+U_{\min } \frac{U_{\min }-U_{\min , \mathrm{t}}}{X-X_{t}}
\end{aligned}
$$

This expression is used in Section 4.2.3 to estimate the pressure above the transition. 
The estimation of the amount of heat transported by the flow in Zones VI and VII comes from the energy equation that can be written:

$$
U \frac{\partial \Delta T_{c}}{\partial x} \simeq-\frac{\partial\left\langle u^{\prime} \theta^{\prime}\right\rangle}{\partial x}-\frac{\partial\left\langle v^{\prime} \theta^{\prime}\right\rangle}{\partial y}
$$

where the molecular diffusion is neglected. Due to the symmetry of the flow with respect to $y=b / 2$, the horizontal turbulent heat flux $\rho C_{p}\left\langle v^{\prime} \theta^{\prime}\right\rangle$ is zero at $y=b / 2$. Considering Equation 8, one can estimate:

$$
\frac{\partial\left\langle v^{\prime} \theta^{\prime}\right\rangle}{\partial y} \simeq-\alpha(x) \frac{2 q_{w}}{\rho C_{p} b}
$$

where the horizontal transport due to the mean flow is neglected as the bulk temperature is supposed to be independent on $y$ in Zones VI and VII. Equation 40 can be written:

$$
\frac{\partial\left\langle u^{\prime} \theta^{\prime}\right\rangle}{\partial x} \simeq-U \frac{\partial \Delta T_{c}}{\partial x}+\alpha \frac{2 q_{w}}{\rho C_{p} b}
$$

From this expression, one can obtain the following order of magnitude :

$$
U \Delta T_{c}+\left\langle u^{\prime} \theta^{\prime}\right\rangle \simeq \alpha \frac{2 q_{w}}{\rho C_{p} b}\left(x-x_{t}\right)
$$

The upper part of the channel is too short to exhibit a clear behaviour of the

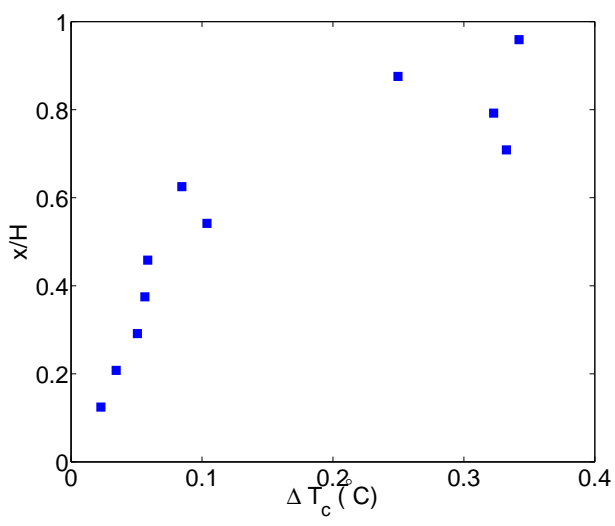

Figure 9: Temperature profile measured at the center of the channel.

temperature in the centre $\Delta T_{c}$ shown on Figure 9. According to Equation 41, a constant or a decreasing $\Delta T_{c}$ in the upper part, means that the vertical turbulent heat flux $\left\langle u^{\prime} \theta^{\prime}\right\rangle$ increases in the Zones VI and VII as the fluid rises. 


\section{Discussions}

The partition of the domain proposed in this study results from the modelling of the main physical phenomena driving the flow and the validation comes from the comparison with experimental data. Two points are discussed below.

These results confirm the scenario for the transition given in the companion paper. In the near-wall region, heat transfer remains laminar in a TBsL along the walls. In the bottom part, the flow is similar to the one that results from a laminar thermally driven flow along a vertical flat plate, in particular, the velocity increases along the heated plate due to buoyant effect $\left(U_{I} \propto X^{3 / 5}\right.$, Eq. $\left.4 \mathrm{~b}\right)$.

Considering a two-dimensional flow, this leads to a decrease in the velocity at center of the channel and according to the analysis of Zone IV, this decrease is linear with respect to $x: U_{I V}=U_{\min } \propto X$ (Eq. 17a). It is then observed that the dynamic of the flow at the center of the channel drives the dynamic of the flow outside the TBsL. Indeed, a best linear fit for $U_{\max }$ in the bottom part is shown in Figure 4. The good agreement with the experimental data suggests that $U_{\max }-U_{\text {inlet }} \propto U_{\text {min }}-U_{\text {inlet }} \propto X$ which means that these two quantities can be scaled by the same quantity. If we suppose that $U_{\text {min }}-U_{\text {inlet }}$ is the relevant velocity scale in the Zones II, III and IV, the flow exhibits two different velocity scales in the near-wall region where $U_{I} \propto X^{3 / 5}$ (Eq. 4b) and in the bulk flow where $U_{\text {min }}-U_{\text {inlet }} \propto X$ which is consistent with the analysis done by $\mathrm{Ng}$ et al. [13] for an infinite channel with isothermal plates.

Supposing that the Reynolds stress follows the same scale outside the TBsL, Equations 30a and 34 leads to :

$$
\begin{array}{r}
\delta_{I I} \propto X \\
\delta_{I I I} \propto X .
\end{array}
$$

These linear evolutions are consistent with the experimental data (see Figure 5 in the companion paper). Moreover, Equation 30b leads to a linear evolution for the temperature in Zone II:

$$
\Delta T_{I I} \propto X
$$


But the experimental temperature profile close to the wall is too coarse and this last prediction cannot be checked from the experimental data.

Following the scenario describes in the companion paper, the transition is triggered by the junctions of the left and right shear layers. Indeed, at this point, the decrease in $U_{\min }$ is counteracted by the momentum diffusion, therefore $\frac{\partial U_{\min }}{\partial x}$, as well as $\frac{\partial U_{\max }}{\partial x}$ approach zero at $x=x_{t}$. By continuity, one expects that the decrease in the velocity peak leads to a decrease in the velocity scale inside the TBsL. Therefore the energy conservation $\rho C_{p} U_{I} \Delta T_{I} \delta_{I}=q_{w} x$ that holds in the bottom part is no longer valid at the transition point. As Equations 4 lead to $U_{I} \Delta T_{I} \delta_{I} \propto U_{I}^{5 / 3}$, close to the transition, a decrease in the velocity inside the TBsL leads to a decrease in the heat transported in this zone. As the wall heat flux is constant all along the plate, energy conservation implies that a part of the energy contained inside the TBsL has to be ejected from this sublayer. This mecanism is modelled by Equation 8. As a consequence, in the top part of the channel, a turbulent transport occurs between this TBsL and the bulk flow. However, the assumptions leading to the wall temperature profile in the top part (Eq. 12a, Fig. 2) are consistent with a laminar TBsL. From a physical point of view, the horizontal heat transfer in Zone $\mathrm{V}$ is dominated by conduction and outside this zone, velocity fluctuations are strong enough to pull hot fluid out of the TBsL leading to an efficient turbulent heat transfer.

Concerning the pressure, Figure 6 shows that the pressure force is an opposing force all along the channel. However, the exit to entry pressure difference is mainly due to the buoyant term in the top part of the channel. Indeed, Figure 6 shows that the pressure increase in the bottom part of the channel is weak and Equation 28b can be approximated as follows:

$$
\frac{P_{g}}{\rho}(H)-\frac{P_{g}}{\rho}\left(x_{t}\right) \simeq g \beta \Delta T_{c, t}\left(H-x_{t}\right)
$$

If the pressure difference in the bottom part is neglected with respect to the one in the top part, the order of magnitude of the pressure difference in the channel is roughly given by:

$$
\frac{P_{g}}{\rho}(H)-\frac{P_{g}}{\rho}(0) \simeq g \beta \Delta T_{c, t}\left(H-x_{t}\right)
$$


Supposing that the surrounding of the channel is at rest and thermally stratified with a temperature difference of $\Delta T_{\text {surr }}$ over the height of the channel, the corresponding pressure difference is given by:

$$
\frac{\Delta P_{g, \text { surr }}}{\rho}=g \beta \Delta T_{\text {surr }} H
$$

Therefore, a thermal stratification of the surrounding of the channel with a temperature difference of $\Delta T_{\text {surr }}=\Delta T_{c, t} \frac{H-x_{t}}{H} \approx 0.1^{\circ} \mathrm{C}$ between $x=H$ and $x=0$, leads to the same order of magnitude for the pressure difference inside and outside the channel. In the experimental study the thermal stratification of the surrounding is not known accurately so we can suppose that the pressure at the exit is set by the pressure of the surrounding at the same level. Therefore, a larger external stratification of the surrounding would increase the pressure difference along the channel which would increase the opposing pressure force in the channel. However, this hypothesis has to be assessed by other investigations with a better control of the stratification of the surrounding.

\section{Conclusion}

In this study, the transition to a turbulent heat transfer in a vertical channel with wall heating at a modified Rayleigh number of $6.710^{7}$ is analysed through a scaling analysis. This analysis leads to analytical expressions that can be used in simplified modelling of such a system. In particular, the wall temperature is found to follow an $x^{1 / 5}$ law below the transition whereas it is proportional to $(a-b x)^{1 / 5}(a$ and $b>0)$ in the upper part. Due to turbulent heat transfer in the upper part, the fluid is heated in the center of the channel which leads to a large increase in the driving pressure. As the pressure difference along the channel is of the same order of magnitude as the one that results from a possible external stratification, more researches are needed to find whether the pressure at the exit is set by the external stratification or not.

\section{Acknowledgements}

This research was funded by the French National Research Agency in the framework of the project $n^{\circ}$ ANR-08-JCJC-0018-01 and the National Agency of Energy Management ADEME-0705C0076. 


\section{Appendix A. Analytical expression of the pressure in Zone IV}

In the central zone IV,

$$
\begin{aligned}
U_{I V} & =U_{\min }(x) \\
V_{I V} & =\left(\frac{b}{2}-y\right) \frac{\partial U_{\min }}{\partial x}
\end{aligned}
$$

The momentum equation along $x$ leads to:

$$
\begin{aligned}
\frac{P_{g I V}}{\rho}+\left\langle u^{\prime 2}\right\rangle-\left[\frac{P_{g I V}}{\rho}+\left\langle u^{\prime 2}\right\rangle\right]_{\text {inlet }} & =\frac{U_{\text {inlet }}^{2}-U_{\text {min }}^{2}}{2} \\
& +\nu\left(\frac{\partial U_{\min }}{\partial x}(x)-\frac{\partial U_{\min }}{\partial x}(0)\right)(A
\end{aligned}
$$

where $U_{\text {inlet }}=U_{\min }(x=0)$. The $y$-momentum gives:

$$
\left(\frac{b}{2}-y\right)\left(U_{\min } \frac{\partial^{2} U_{\min }}{\partial x^{2}}-\left(\frac{\partial U_{\min }}{\partial x}\right)^{2}\right)=-\frac{\partial}{\partial y}\left[\frac{P_{g I V}}{\rho}+\left\langle u^{\prime 2}\right\rangle\right]_{\text {inlet }}+\frac{\partial\left(\left\langle u^{\prime 2}\right\rangle-\left\langle v^{\prime 2}\right\rangle\right)}{\partial y}
$$

where the subscript inlet denotes quantities evaluated at $x=0$. Due to the symmetry of the mean flow with respect to the plane $y=b / 2$,

$$
\frac{\partial\left\langle u^{\prime 2}\right\rangle}{\partial y}\left(x, y=\frac{b}{2}\right)=\frac{\partial\left\langle v^{\prime 2}\right\rangle}{\partial y}\left(x, y=\frac{b}{2}\right)=0 .
$$

We suppose that these terms remains negligible in Zone IV. Therefore, as the quantities at the inlet depends on $y$ and $U_{\text {min }}$ depends on $x$, one can write:

$$
\begin{aligned}
U_{\min } \frac{\partial^{2} U_{\min }}{\partial x^{2}}-\left(\frac{\partial U_{\min }}{\partial x}\right)^{2} & =2 A A \\
{\left[\left(\frac{P_{g I V}}{\rho}+\left\langle u^{\prime 2}\right\rangle\right)_{\text {inlet }}\right]_{b / 2}^{y} } & =A A\left(\frac{b}{2}-y\right)^{2}
\end{aligned}
$$

where $A A$ is constant. To solve Equation A.2, it is supposed that $U_{\min } \simeq X^{n}$. Then, first equation leads to $n=1$ which implies

$$
-\left(\frac{\partial U_{\min }}{\partial x}\right)^{2}=2 A A=-A_{m}^{2} \leq 0
$$


where $A_{m}$ is introduced to take into account for the negative sign of $A A$.

So in the central region, one obtains:

$$
\begin{aligned}
& U_{I V}=U_{\min }(x)=-A_{m} x+U_{\text {inlet }} \\
& V_{I V}=-A_{m}\left(\frac{b}{2}-y\right)
\end{aligned}
$$

Equation A.3 gives the pressure profile at the inlet:

$$
\left[\left(\frac{P_{g I V}}{\rho}+\left\langle u^{\prime 2}\right\rangle\right)_{\text {inlet }}\right]_{b / 2}^{y}=-\frac{A_{m}^{2}}{2}\left(\frac{b}{2}-y\right)^{2}
$$

Then from Equation A.1, one obtains the pressure in Zone IV:

$$
\begin{aligned}
\frac{P_{g I V}}{\rho}+\left\langle u^{\prime 2}\right\rangle & =\left(\frac{P_{g I V}}{\rho}+\left\langle u^{\prime 2}\right\rangle\right)(x=0, y)+\frac{U_{\text {inlet }}^{2}-U_{\text {min }}^{2}}{2} \\
& =\left(\frac{P_{g I V}}{\rho}+\left\langle u^{\prime 2}\right\rangle\right)(0, b / 2)-A_{m} x\left(A_{m} x-2 U_{\text {inlet }}\right)-\frac{A_{m}^{2}}{2}\left(\frac{b}{2}-y\right)^{2}
\end{aligned}
$$

In particular, at $x=0$, it implies:

$$
\left(\frac{P_{g I V}}{\rho}+\left\langle u^{\prime 2}\right\rangle\right)(x=0, y)=\left(\frac{P_{g I V}}{\rho}+\left\langle u^{\prime 2}\right\rangle\right)(0, b / 2)-\frac{A_{m}^{2}}{2}\left(\frac{b}{2}-y\right)^{2}
$$

\section{Appendix B. Analytical expression of the pressure gradient below transition}

As the flow is symmetrical with respect to $z=0.5 l$, one can suppose that rms fluctuations are independent of $z$ and Equation $2 d$ leads to a zero pressure gradient in the z-direction. Then by using Equations 18, one obtains:

$$
\frac{P_{g}}{\rho}=-\left\langle v^{\prime 2}\right\rangle+g(x)
$$

The unknown function $g$ is deduced from Equation $17 \mathrm{c}$ :

$$
\begin{aligned}
g(x) & =\frac{P_{g}}{\rho}(x, b / 2)+\left\langle v^{\prime 2}\right\rangle(x, b / 2) \\
& =-\left\langle u^{\prime 2}\right\rangle(x, b / 2)+\left\langle v^{\prime 2}\right\rangle(x, b / 2)+\frac{U_{\text {inlet }}^{2}-U_{\text {min }}^{2}}{2}+\left(\frac{P_{g I V}}{\rho}+\left\langle u^{\prime 2}\right\rangle\right)(x=0, y)
\end{aligned}
$$


So, the pressure gradient in the streamwise direction is:

$$
\begin{aligned}
\frac{\partial}{\partial x}\left[\frac{P_{g}}{\rho}+\left\langle u^{\prime 2}\right\rangle\right]= & -U_{\min } \frac{\partial U_{\min }}{\partial x} \\
& +\frac{\partial}{\partial x}\left[\left\langle u^{\prime 2}\right\rangle-\left\langle v^{\prime 2}\right\rangle\right](x, y)-\frac{\partial}{\partial x}\left[\left\langle u^{\prime 2}\right\rangle-\left\langle v^{\prime 2}\right\rangle\right](x, b / 2)
\end{aligned}
$$

[1] C. Daverat, Y. Li, H. Pabiou, C. Ménézo, S. Xin, Transition to turbulent heat transfer in heated vertical channel - Experimental analysis, International Journal of Thermal Sciences 111 (2017) 321-329.

[2] C. Daverat, H. Pabiou, C. Ménézo, H. Bouia, S. Xin, Experimental investigation of turbulent natural convection in a vertical water channel with symmetric heating: Flow and heat transfer, Experimental Thermal and Fluid Science 44 (2013) 182-193.

[3] E. Sparrow, J. Gregg, Laminar Free Convection From a Vertical Plate With Uniform Surface Heat Flux, Transaction of the ASME (1956) 435440.

[4] W. Lin, S. W. Armfield, Unsteady natural convection on an evenly heated vertical plate for Prandtl number $\operatorname{Pr}<1$, Physical Review E 72 (6) (2005) 066309.

[5] W. Lin, S. W. Armfield, J. C. Patterson, C. Lei, Prandtl number scaling of unsteady natural convection boundary layers for $\operatorname{Pr}>1$ fluids under isothermal heating, Physical Review E 79 (6) (2009) 066313.

[6] R. Khanal, C. Lei, A scaling investigation of the laminar convective flow in a solar chimney for natural ventilation, International Journal of Heat and Fluid Flow 45 (2014) 98-108. doi:10.1016/j.ijheatfluidflow.2013.11.002.

[7] A. Bar-Cohen, W. M. Rohsenow, Thermally optimum spacing of vertical, natural convection cooled, parallel plates, Journal of Heat Transfer 106 (1) (1984) 116-123.

[8] N. Sobel, F. Landis, W. Mueller, Natural convection heat transfer in short vertical channels including the effects of stagger, Proceedings 3rd International Heat Transfer Conference 2 (1966) 121-125. 
[9] D. Lohse, K.-Q. Xia, Small-scale properties of turbulent RayleighBénard convection, Annual Review of Fluid Mechanics 42 (2010) 335364.

[10] F. Chilla, J. Schumacher, New perspectives in turbulent RayleighBénard convection, The European Physical Journal E: Soft Matter and Biological Physics 35 (7) (2012) 1-25.

[11] S. Grossmann, D. Lohse, Scaling in thermal convection: a unifying theory, Journal of Fluid Mechanics 407 (2000) 27-56.

[12] S. Grossmann, D. Lohse, Fluctuations in turbulent Rayleigh-Bénard convection: the role of plumes, Physics of Fluids (1994-present) 16 (12) (2004) 4462-4472.

[13] C. S. Ng, A. Ooi, D. Lohse, D. Chung, Vertical natural convection: application of the unifying theory of thermal convection, Journal of Fluid Mechanics 764 (2015) 349-361. 